\title{
Publisher's Note: Graph theory data for topological quantum chemistry [Phys. Rev. E 96, 023310 (2017)]
}

M. G. Vergniory, L. Elcoro, Zhijun Wang, Jennifer Cano, C. Felser, M. I. Aroyo, B. Andrei Bernevig, and Barry Bradlyn ${ }^{(0)}$

(Q) (Received 2 June 2020; published 11 June 2020)

DOI: 10.1103/PhysRevE.101.069902

This paper was published online on 28 August 2017 with an error in the Acknowledgment section. The last sentence of the first paragraph of the Acknowledgment section should read as "The ab initio work was funded by the DOE Grant No. de-sc0016239. The development of the practical part of the theory, tables, and some of the code development was funded by a Simons Investigator Award, the Packard Foundation, and the Schmidt Fund for Innovative Research." The Acknowledgments have been corrected as of 4 June 2020. The Acknowledgments are incorrect in the printed version of the journal. 